\title{
Retroperitoneal Cystic Lymphangioma: About A Case and Review of The Literature
}

\author{
Graiouid M, Bai W, Messian G, Jandou I, Larach Y, Doumer A, Elafifi M, Abdi M, Dakir M, \\ Debbagh A, and Aboutaieb R.
}

\begin{abstract}
Cystic lymphangioma is a rare benign malformative tumor of lymphatic vessels in various locations.

We report the observation of a 62-year-old man who had been consulting for major abdominal distention for 5 years. The clinical examination found a very distended abdomen with collateral venous circulation.

The imaging was in favor of a cystic retro-peritoneal image. Surgical exploration showed a retroperitoneal cystic mass. Anatomo-pathological examination concluded that retroperitoneal cystic lymphangioma.
\end{abstract}

Index Terms - Retroperitoneal cystic lymphangioma, diagnosis, treatment.

\section{INTRODUCTION}

Retroperitoneal localization is less common compared to mesenteric. His clinical presentation is polymorphic. Diagnosis is evoked by imaging but requires histological confirmation. The treatment of choice is surgical. Retroperitoneal cystic lymphangioma represents a rare benign tumor whose clinical presentation is polymorphic. Diagnosis is evoked by imaging but requires histological confirmation. The treatment of choice is surgical.

\section{OBSERVATION}

A 62-year-old man, with no particular pathological history, was seen for a progressive abdominal distension for 5 years complicating with transit trouble three months before the consultation.

Clinical examination revealed significant abdominal distension; an unfolding of the umbilicus and a collateral venous circulation (Figure 1), the palpation objectified a fluid mass occupying the whole abdomen, mobile with respect to the superficial planes.

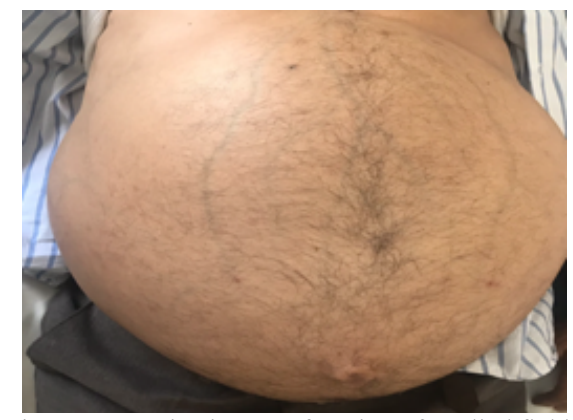

Fig. 1. Magnetization as a function of applied field.

Published on December 20, 2019
The rest of the clinical examination was normal. The biological assessment was correct.

The abdominal ultrasound showed a huge right retroperitoneal fluid mass pushing the right kidney forward and to the left side and extending to the pelvis without detectable lymphadenopathy. The abdominal CT showed the presence of a partitioned abdominal-pelvic cystic mass with heterogeneous enhancement, pushing back the colonic framework and the small bowels anteriorly and the inferior vena cava and the aorta behind and the kidney forward and to the left (Figure 2).

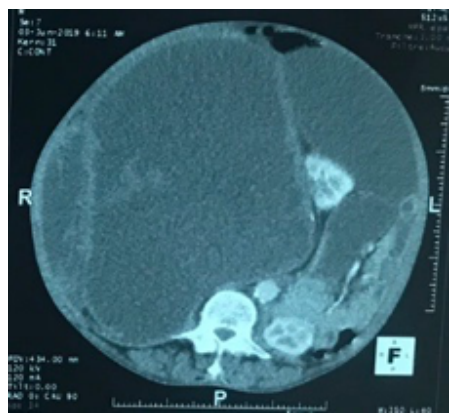

Fig. 2. Magnetization as a function of applied field.

The surgical exploration found a voluminous multiperitoneal lobulated cystic mass containing a citric fluid; complete resection was performed. The anatomopathological study had concluded a retroperitoneal cystic lymphangioma.

\section{DISCUSSION}

Cystic lymphangiomas are rare benign tumors $[1,2]$ seen mainly in children [3]. In adults, they represent only $7 \%$ of abdominal cysts [4] Two theories have been proposed for origin of these cystic lymphangioma. The first suggests an acquired origin resulting from an obstruction of the lymphatic vessels as a result of inflammation, trauma or degeneration [5]. The second speaks of a malformative origin. The most frequently affected areas are the subcutaneous tissues of the neck (about 75\%) and underarms (about 15\%). The mediastinal and abdominal locations are much rarer, about $10 \%$ of cases. Retroperitoneal localization is less common than mesenteric localization [6].

The clinical manifestations of retroperitoneal cystic lymphangioma are highly polymorphic [7]. They are related to tumor volume or complication, ranging from an often asymptomatic mass in adults to acute abdominal pain or even complications such as rupture, intra-cystic haemorrhage, occlusion, torsion, compression or infiltration 
of vital structures. A large tumor volume usually causes abdominal pain, the most common symptom [8].

No sign is specific and it is the imaging assessment that will guide the diagnosis. Ultrasound is the most useful examination initially. It typically shows a plain or multilocular fluid tumor with thin septa that is well-limited [9]. The content of cysts, which is often transonic, can become echogenic with intra-cystic haemorrhage or even contain some calcifications [9]. The CT allows to study the density of the tumor and to evaluate more precisely the relations with the neighboring organs and to differentiate the intra and retroperitoneal lymphangiomas. The MRI also allows an accurate diagnosis, appreciating very well the perivascular extension of the lesion [5].

Diagnostic evidence of cystic lymphangioma is provided by pathological examination. Histologically, retroperitoneal lymphangioma consists of cavities lined by an endothelium resting on fibrous tissue containing lymphocytic islets and sometimes smooth muscle fibers. Inflammation and haemorrhage often cause reshaping with disappearance of the endothelium, appearance of fibrin deposits, sometimes making the histological diagnosis impossible on simple biopsies [7].

The therapeutic indication must take into account the benign nature of the tumor, but also and especially complications often revealing lymphangioma. Surgical excision is the classic attitude; it must be as complete as possible, thus avoiding recurrences. [10], while remaining conservative for other organs, given the benign nature of lymphangioma.

\section{CONCLUSION}

Retroperitoneal cystic lymphangioma is a rare entity that does not always have a classical appearance. The clinical symptomatology is nonspecific. Diagnosis is suspected on imaging and confirmed only by histology. The treatment of choice is complete surgical excision.

\section{REFERENCES}

[1] Zekri B, Mouaqit O, Affari O, Ifrine L, El Malki HO, Mohsine R, Belkouchi A. Lymphangiome kystique rétropéritonéal. J Afr Hépatol Gastroentérol. $2010 ; 4: 260-262$.

[2] Losanoff JE, Richman BW,El-Sherif A. Mesenteric cystic lymphogioma. J Am Coll Surg. 2003; 196(4): 598-603.

[3] DEBAT ZOGUEREH D., TARUNDEGA U.N., PROVENDIER B. : Une volumineuse masse rétropéritonéale chez un adulte. La revue de la médecine interne, $2003 ; 24: 202-203$

[4] KABLY A., MOUMEN M., RAISSOUNI N. : Le lymphangiome kystique du mésentère et de l'épiploon. A propos de 2 cas. Gynécologie obstétrique et fertilité, $2003 ; 31: 136-138$.

[5] BONHOMME A., BROEDERS A., OYEN R.H. : Cystic lymphangioma of the retroperitoneum. Clinical radiology, $2000 ; 156-$ 158.

[6] Zoguéreh DD, N'Tarundenga U, Provendier B, Gazaigne J. Une volumineuse masse rétropéritonéale chez un adulte. Rev Med Interne. 2003; 24(3):202-203

[7] T, Bühler L, Chardot C, Morel P. Le traitement chirurgical du lymphangiome kystique abdominal chez l'adulte et chez l'enfant. J Chir (Paris). 2008; 145(3):238-243.

[8] Ahmed Saadi, Haroun Ayed, Omar Karray, Walid Kerkeni, Abderrazak Bouzouita1, Mohamed Cherif, Riadh Ben Slama, Amine Derouiche, Mohamed Chebil. Retroperitoneal cystic lymphangioma: about 5 cases and review of the literature Pan African Medical Journal. 2016; 25:73.
[9] Rifki Jai S, Adraoui J, Khaiz D, Chehad F, Lakhloufi A, Bouzidi A. Le lymphangiome kystique rétropéritonéal. Prog Urol. 2004; 14(4):548-550.

[10] RIFKI JAI S., ADRAOUI J., KHAIZ D., CHIHAB F., LAKHLOUFI A., BOUZIDI A., SIWANE A., KADIRI R. Prog Urol, 2004, 14, 548550 . 